DOI: https://doi.org/10.46296/ig.v4i7.0019

\title{
DISEÑO Y EVALUACIÓN DE UN PROTOTIPO PARA VIVIENDA DE INTERÉS SOCIAL CON MAMPOSTERÍA CONFINADA EN LADRILLO
}

\section{DESIGN AND EVALUATION OF A PROTOTYPE FOR HOUSING OF SOCIAL INTEREST WITH MASONRY CONFINED IN BRICK}

\author{
Intriago-Plaza Renato ${ }^{1 *}$; Palma-Zambrano Willians²; Mieles-Bravo Yordy ${ }^{3}$ \\ ${ }^{1}$ Maestrante del Instituto de Posgrado de la Universidad Técnica de Manabí, UTM. \\ Portoviejo, Ecuador. \\ ${ }^{2,3}$ Docente del Instituto de Posgrado de la Universidad Técnica de Manabí, UTM. \\ Portoviejo, Ecuador.
}

*Correo: intriago8381@utm.edu.ec

\begin{abstract}
Resumen
Las condiciones actuales de hábitat en el mundo se ven seriamente afectadas por la sobrepoblación y los permanentes flujos de movilidad territorial, generando la necesidad de construir viviendas con diseños ecológicos, adaptables y de bajo costo. En este sentido, la presente investigación permitió realizar el diseño y análisis de un prototipo de vivienda de interés social con muros de mampostería confinada en ladrillo. Cabe señalar que la vivienda ocupa un espacio de $82 \mathrm{~m}^{2}$, misma que ha sido diseñada para su construcción en un terreno de $200 \mathrm{~m}^{2}$, ubicado en la ciudad de Manta, Ecuador. Además del diseño estructural de la vivienda, se realizó una investigación con enfoque cuantitativo, puesto que se realizaron 25 encuestas dirigidas a los directivos de constructoras y ferreterías de la provincia de Manabí, en las que se pudo obtener información relacionada con las tipologías de viviendas de interés social y los sistemas constructivos empleados con el respectivo análisis comparativo de costos y el mercado de viviendas en función de la oferta y demanda; por su parte, el precio unitario de los materiales, equipos y mano de obra requeridos en la construcción del sistema estructural propuesto fue obtenido de casas distribuidoras de materiales y las tablas salariales, respectivamente. Los resultados reflejan que la vivienda diseñada y evaluada en la presente investigación consta de un nivel; en el que se encuentra la sala, comedor, cocina, garaje y dormitorios. El costo total requerido para ejecutar la construcción del diseño propuesto equivale a \$30075,05 dólares americanos incluido IVA, representando un total de 75,19 sueldos básicos. Se estableció que la vivienda de interés social con mampostería confinada de ladrillo presenta un sistema estructural favorable para su aplicación en el medio local de Manta, y de la misma forma puede extrapolarse a otras localidades del país.
\end{abstract}

Palabras clave: viviendas sociales, diseño, estructura, mampostería, evaluación económica.

\begin{abstract}
Current habitat conditions in the world are seriously affected by overpopulation and permanent flows of territorial mobility, generating the need to build houses with ecological, adaptable and low-cost designs. In this sense, the present investigation allowed to carry out the design and analysis of a social interest housing prototype with masonry walls confined in brick. It should be noted that the house occupies a space of $82 \mathrm{~m}^{2}$, which has been designed for construction on a $200 \mathrm{~m}^{2}$ plot of land, located in the city of Manta, Ecuador. In addition to the structural design of
\end{abstract}

Información del manuscrito:

Fecha de recepción: 01 de diciembre de 2020.

Fecha de aceptación: 08 de enero de 2021.

Fecha de publicación: 11 de enero de 2021. 
the house, an investigation was carried out with a quantitative approach, since 25 surveys were carried out directed to the directors of construction companies and hardware stores in the province of Manabí, in which information related to the types of homes of social interest and the construction systems used with the respective comparative analysis of costs and the housing market based on supply and demand. On the other hand, the unit price of the materials, equipment and labor required in the construction of the proposed structural system was obtained from material distribution houses and the salary tables, respectively. The results reflect that the house designed and evaluated in the present investigation consists of one level; in which the living room, dining room, kitchen, garage and bedrooms are located. The total cost required to execute the construction of the proposed design is equivalent to US \$30,075.05 including VAT, representing a total of 75.19 basic salaries. It was established that low-income housing with confined brick masonry presents a favorable structural system for its application in the local environment of Manta, and in the same way it can be extrapolated to other localities in the country.

Keywords: social housing, design, structure, masonry, economic evaluation.

\section{Introducción}

El crecimiento poblacional se mantiene en auge permanente durante los últimos años, de modo que en Latinoamérica la población del año 1950 era 69 millones de personas y hasta el 2010 aumentó en un $600 \%$. Esto sugiere que la migración se ha expandido de forma masiva y más aún los flujos migratorios desde las zonas rurales hacia zonas consideradas urbanas. Según el Banco Interamericano de Desarrollo (BID), aproximadamente 160 millones de personas habitan en barrios informales, lo cual aumenta el costo de la tierra y, por lo tanto, la accesibilidad de un terreno o vivienda se reduce para personas de escasos recursos. En esta región existe un déficit de vivienda estimado en términos cuantitativos (número de familias sin vivienda) en más de 28 millones; mientras que en términos cualitativos (viviendas con deficiencias: materialidad, espacio y servicios) se refleja un déficit superior a los 25 millones (BID, 2014).

Ecuador no es la excepción en la problemática de la vivienda, puesto que la población está en franco aumento y se prevé que para el año 2050 , en este país sudamericano habiten 23 millones de personas. Esto conlleva a una mayor demanda habitacional, que evidentemente no ha podido ser resuelta por los gobiernos de turno. Se estima que en el Ecuador el déficit habitacional de las viviendas supera el $75 \%$ (INEC, 2010).

Las condiciones actuales del mundo se ven afectadas por la sobrepoblación y los constantes flujos de movilidad territorial, 
ocasionando la necesidad de construir viviendas en serie con diseños ecológicos, adaptables y de bajo costo (Ocampo, 2017). En este sentido, las viviendas de interés social (VIS) constituyen un aporte importante para abordar esta problemática, de manera que las viviendas diseñadas en función de las necesidades y requerimientos de los usuarios, así como su entorno con la ciudad, son una propuesta viable para solucionar esta necesidad básica detectada en todos los países latinoamericanos. No obstante, en nuestro medio predominan los modelos $y$ propuestas de viviendas de interés social con enfoque asociado principalmente al cumplimiento de parámetros cuantitativos, que en la mayoría de los casos no consideran aspectos como la relación de la vivienda con la calidad del diseño, la relación con el entorno-ambiente y los aspectos técnicos de funcionalidad asociados con las expectativas de los usuarios (PérezPérez, 2016).

Por otra parte, la sociedad ecuatoriana actualmente se encuentra acostumbrada a las edificaciones convencionales, es decir, sistemas convencionales basados en la implementación de elementos estructurales, tales como; columnas, vigas y losas, sean estas de hormigón armado o estructuras metálicas, donde los costos dependen del análisis que se obtenga en función de los volúmenes de producción. Por tanto, se hace referencia a la implementación de diseños con el mismo tipo de estructura con diferentes sistemas de cerramiento, respetando las condiciones y normas ecuatorianas de construcción (Cárdenas \& Macancela, 2018).

La VIS debe cumplir con la necesidad básica de vivienda que presenta el Ecuador, pero también debe garantizar espacios funcionales que brinden condiciones óptimas para el desarrollo psicológico y social, además, debe promover la sostenibilidad de los recursos en función de costos accesibles (Bamba, 2016). Adicionalmente, para cumplir con las exigencias en el volumen de construcción y con los costos reducidos, estas viviendas deben construirse en el menor tiempo posible; es por esta razón, que previo a la construcción de las VIS 
deben evaluarse los costos totales que permitan determinar la factibilidad del diseño (Carrillo et al., 2015).

Por consiguiente, el objetivo principal de la investigación consiste en realizar el diseño y análisis de un prototipo de vivienda de interés social con muros de mampostería confinada en ladrillos.

\section{Metodología}

\subsection{Tipo de investigación}

La investigación implementada en este estudio tuvo un enfoque cuantitativo, puesto que se aplicaron encuestas dirigidas a los directivos o administradores de ferreterías o/y franquiciados de materiales de construcción de la provincia de Manabí, Ecuador; en aras de conocer el tipo de materiales y los costos unitarios de los materiales, equipos y mano de obra requerida en la construcción de una vivienda de interés social de tipo mampostería confinada en ladrillos.

\subsection{Tareas de investigación}

En concordancia con lo antes manifestado, se plantearon las siguientes tareas de investigación:
- Revisión de la literatura con aportes en el diseño, construcción y evaluación económica de las viviendas de interés social.

- Determinar los elementos clave que forman parte del diseño de una vivienda con mampostería confinada en base a la NEC.

- Diseñar un prototipo de vivienda de interés social con muro de mampostería confinada en ladrillo.

- Determinar los precios unitarios de materiales, equipos y mano de obra, que corresponde al análisis de precios unitarios en función de la moneda oficial del Ecuador (USD), con la finalidad de establecer un presupuesto para la construcción del prototipo de la vivienda de interés social propuesta.

- Analizar el presupuesto requerido para la construcción del diseño mediante un programa informático.

- Analizar las características técnicas del diseño propuesto; comparar los costos obtenidos con estudios realizados en contextos similares en función de 
diseños de viviendas de interés social.

\subsection{Métodos, técnicas y procedimientos de investigación}

En el marco del presente proyecto de investigación se utilizó el método inductivo: histórico-lógico, además del teórico, puesto que se revisó la literatura científica que está vinculada con el diseño, evaluación y construcción de viviendas de interés social. Se aplicó un método cuantitativo (encuestas) para determinar los costos de materiales, equipos y manos de obra en el mercado local a través de fuentes de información primaria, como lo son los administradores de constructoras y ferreterías de la provincia de Manabí, Ecuador. Posteriormente, se realizó un análisis estructural del prototipo de vivienda de interés social con muros de mampostería confinada en ladrillo mediante la técnica de modelación matemática y la estadística descriptiva. Una vez obtenido el diseño estructural de la VIS, se evaluaron los costos de producción del sistema mediante el cálculo de precio unitario en el software InterPro, aplicando el método analítico.

\subsection{Población y muestra de la investigación}

La población que se ha escogido para la investigación es el barrio "Jesús de Nazareth" de la ciudad de Manta, debido a que este sector es un lugar estratégico donde se evidencia el crecimiento poblacional de la ciudad. Tomando en cuenta las condiciones de este sector, se realizó el diseño de la vivienda de interés social en $82 \mathrm{~m}^{2}$ de construcción para un terreno de 200 $\mathrm{m}^{2}$, en donde se pudo realizar el diseño y análisis de la VIS con muros de mampostería confinada en ladrillo.

Por su parte, en lo referente a recolección de información primaria, se aplicaron encuestas dirigidas a los administradores y directivos de constructoras, ferreterías y casas de distribución de materiales de construcción radicadas en la provincia de Manabí, de donde se pudo obtener información referente al costo unitario de materiales y mano de obra utilizada en la construcción del diseño propuesto. En total se muestrearon 25 constructoras y ferreterías de la provincia de Manabí; debido a que, para los fines de la investigación, no 
es necesario contar con una muestra amplia ni con un diseño muestral exhaustivo que demande un excesivo tiempo de análisis de la información. Adicionalmente, se aplicó un muestreo aleatorio, por cuotas.

\section{Resultados y discusión}

\subsection{Diseño de la vivienda}

En el desarrollo de la investigación se planteó un diseño estructural de la vivienda de interés social con mampostería confinada. Este diseño fue propuesto considerando las normas ecuatorianas de construcción (NEC). investigación consta de un nivel; en el que se encuentra las siguientes dependencias: sala, comedor, cocina, garaje y dormitorios. La altura de la vivienda es de 2,4 metros en la parte baja de la cubierta y 4,4 metros en la parte alta de la misma, por lo que la altura máxima de la vivienda alcanza los 4,40 metros medidos desde el contrapiso. En la figura 1 se presenta un corte longitudinal de la vivienda, con un detalle constructivo tipo sección vertical, mientras que en la figura 2 se muestra la planta arquitectónica de la vivienda de interés social con mampostería confinada en ladrillo.

La vivienda de interés social diseñada y evaluada en la presente

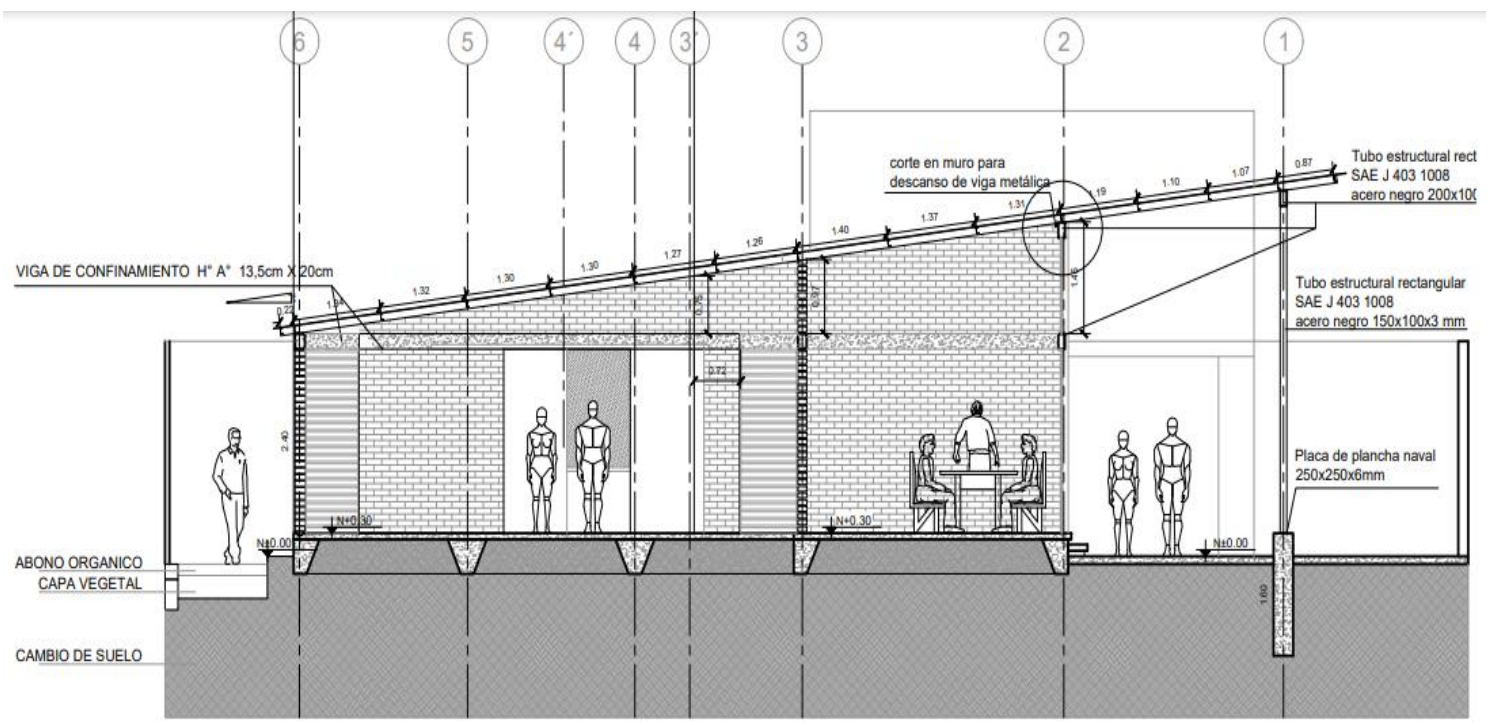

Figura 1. Corte longitudinal de la vivienda de interés social propuesta 


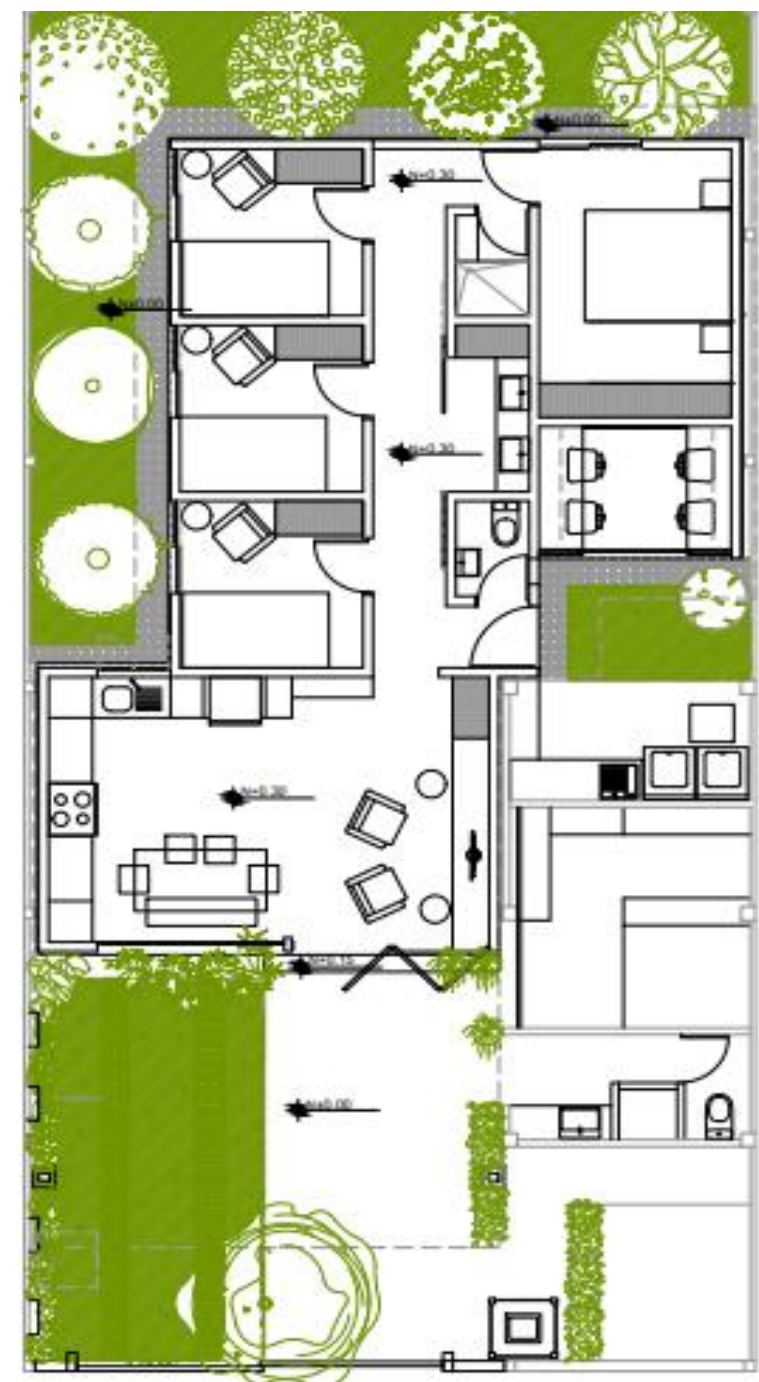

Figura 2. Planta arquitectónica de la vivienda de interés social propuesta

La solución estructural de la vivienda se basa en la conformación de muros de mampostería de ladrillo confinada, asentada sobre riostras de hormigón armado de $135 \mathrm{~mm} \times$ $200 \mathrm{~mm}$, que recorre todo el sistema de muros, esta estructura ha sido diseñada para resistir de forma adecuada las cargas verticales asociadas al peso de la edificación y al uso de la misma, así como otras cargas laterales relacionadas con eventos telúricos y el viento. El sistema propuesto proporciona un alto nivel de flexibilidad para el proyecto arquitectónico.

La cubierta está compuesta por placas de Galvalume con un espesor de $0,40 \mathrm{~mm}$. La cubierta está diseñada para trabajar fundamentalmente en una dirección y estará soportada por correas que 
consisten en perfiles armados de acero estructural.

El sistema para cargas de gravedad de la edificación se compone de muros de mampostería confinada por riostras de hormigón armado. Los muros de mampostería se encuentran proyectados mediante secciones tipo Shell, mientras que las correas de la cubierta se encuentran proyectadas con secciones amadas de acero estructural de sección "G". Por su parte, las riostras de la estructura tienen secciones rectangulares de hormigón armado.

Es fundamental que las edificaciones propuestas en países con alto riesgo sísmico cuenten con estructuras sismorresistentes. Por lo tanto, la presente estructura respeta estos criterios, ajustando el diseño a las normas de construcción pertinentes.
En lo concerniente a las riostras, éstas se diseñaron en base a las sugerencias propuestas en el capítulo 21 del $\mathrm{ACl}$ (American Concrete Institute) y en la norma NEC-SE-HM, en el apartado de la norma ecuatoriana referente al hormigón armado.

Es importante señalar que los muros de mampostería se diseñaron a partir de los lineamientos planteados en la norma NECSE-MP, apartado relacionado con la mampostería, tal como se observa en la figura 3. Los muros de mampostería han sido modelados como elementos tipo "Shell" de sección rectangular.

El material empleado en los muros corresponde a mampostería de resistencia $\quad f^{\prime} m=70 \quad \mathrm{~kg} / \mathrm{cm}^{2}$ (resistencia nominal a la compresión de mampostería).

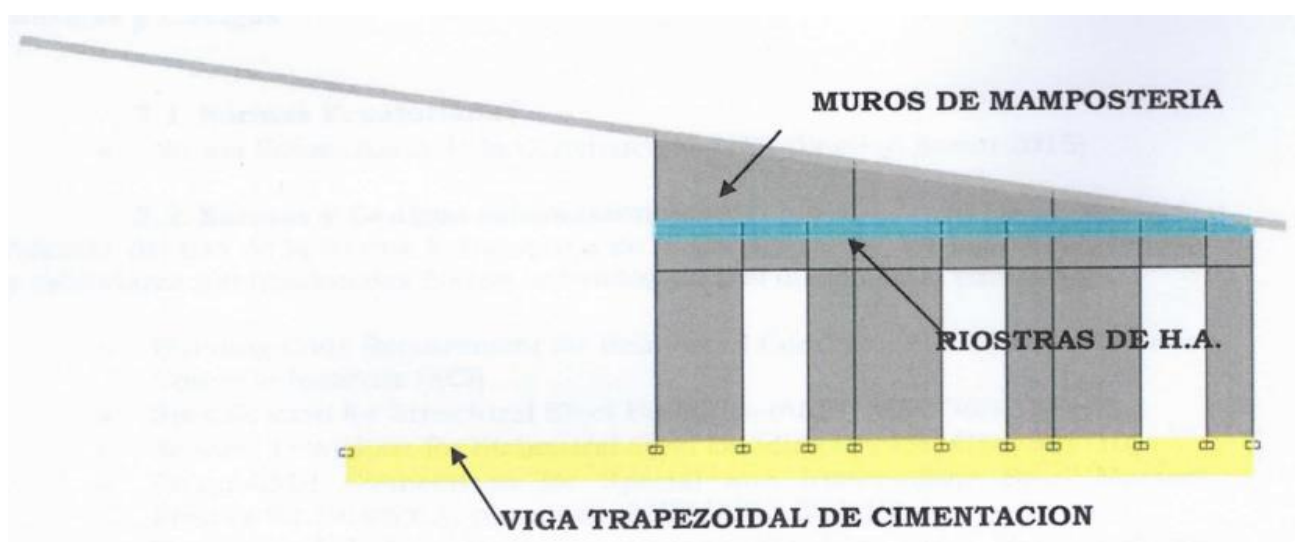

Figura 3. Pórtico de mampostería 
Cabe señalar que los elementos estructurales de acero deben protegerse mediante coberturas a prueba de fuego, de modo que se cumplan los requisitos exigidos en la Norma Ecuatoriana de Construcción (NEC) y el Servicio Ecuatoriano de Normalización (INEN).

En términos mínimos, se proporcionará una resistencia al fuego (Fire Rating) de 2 horas de acuerdo a ASTM E119 (Métodos estandarizados para pruebas de fuego en materiales $y$ construcciones de edificios).

Los elementos estructurales de acero serán protegidos con pintura galvanizada anticorrosiva adecuada para las condiciones del proyecto arquitectónico.

Finalmente, el diseño de las cimentaciones se ha desarrollado en función de las especificaciones detalladas en el Estudio de Mecánica de Suelos realizado por una consultora local. En dependencia de este estudio se determinó que la aceleración de terreno para diseño sísmico tiene un valor de $Z=0,50$; mientras que el suelo se clasifica con la categoría "D", según los parámetros de la
NEC. Adicionalmente, este informe resalta que se utilicen vigas de cimentación tipo "Trapezoidal" Bidireccionales. También se propone que en el fondo de la excavación se realice la siguiente modificación: Piedra bola e=0,75 metros, Material Granular Lastre (mejoramiento tipo MTOP) e $=0,75$ metros, compactado en capas de 0,15 metros al 98\% del Proctor modificado. Cabe especificar que la capacidad admisible del suelo equivale a $19 \mathrm{ton} / \mathrm{m}^{2}$.

\subsection{Presupuesto del diseño de la vivienda}

En la tabla 1 se presenta el presupuesto desglosado por ítems, con el respectivo análisis de precios unitarios, requerido para construir la vivienda de interés social con mampostería confinada en ladrillo. Todos los valores han sido estimados en función de la moneda oficial ecuatoriana (dólar estadounidense) y a partir de los datos aportados por los participantes de las encuestas dirigidas a los representantes de las constructoras, ferreterías y empresas distribuidoras de materiales de construcción radicadas en la provincia de 
Manabí, de manera que se obtuvo

el promedio de los precios

aportados por cada uno de los participantes de la presente

investigación.

Tabla 1.

Análisis de precios unitarios para el sistema estructural propuesto

\begin{tabular}{|c|c|c|c|c|c|c|}
\hline \multicolumn{7}{|c|}{ Presupuesto } \\
\hline Ítem & Código & Descripción & Unidad & Cantidad & $\begin{array}{c}\text { P. } \\
\text { Unitario }\end{array}$ & P. Total \\
\hline 1 & & Preliminares & & & & 892,73 \\
\hline 1.1 & 500148 & Replanteo y nivelación & $\mathrm{m}^{2}$ & 82,53 & 1,14 & 94,08 \\
\hline 1.2 & 500541 & Excavación y desalojo manual & $\mathrm{m}^{3}$ & 37,14 & 12,05 & 447,54 \\
\hline 1.3 & 500 aeo & $\begin{array}{l}\text { Relleno compactado con } \\
\text { mejoramiento tipo MTOP }\end{array}$ & $\mathrm{m}^{3}$ & 23,82 & 14,74 & 351,11 \\
\hline 2 & & $\begin{array}{l}\text { Estructura cimentación - estructura } \\
\text { metálica }\end{array}$ & & & & 7712,44 \\
\hline 2.1 & 500503 & 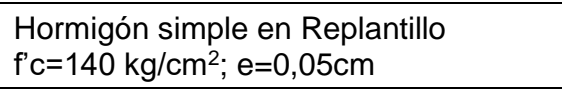 & $\mathrm{m}^{3}$ & 1,305 & 189,49 & 247,28 \\
\hline 2.2 & 500507 & $\begin{array}{l}\text { Hormigón en viga de cimentación de } \\
\text { h.s f }{ }^{\prime} \mathrm{c}=210 \mathrm{~kg} / \mathrm{cm}^{2}\end{array}$ & $\mathrm{~m}^{3}$ & 13,32 & 223,42 & 2975,95 \\
\hline 2.3 & 500586 & $\begin{array}{l}\text { Hormigón en viga para losa alivianada } \\
\text { de } h . s f^{\prime} \mathrm{c}=240 \mathrm{~kg} / \mathrm{cm}^{2}\end{array}$ & $\mathrm{~m}^{3}$ & 2,3 & 218,94 & 503,56 \\
\hline 2.4 & 500015 & Acero de refuerzo $\mathrm{fy}=4200 \mathrm{~kg} / \mathrm{cm} 2$ & $\mathrm{~kg}$ & 926,54 & 2,15 & 1992,06 \\
\hline 2.5 & 500544 & $\begin{array}{l}\text { Mesones de hormigón armado } \\
f^{\prime} c=210 \mathrm{~kg} / \mathrm{cm} 2 \mathrm{e}=7 \mathrm{~cm}\end{array}$ & $\mathrm{~m}$ & 8,6 & 33,26 & 286,04 \\
\hline 2.6 & 500634 & $\begin{array}{l}\text { Contrapiso de hormigón de } \\
f^{\prime} \mathrm{c}=210 \mathrm{~kg} / \mathrm{cm} 2 \mathrm{e}=7 \mathrm{~cm} \text { incluye malla } \\
\text { electrosoldada }\end{array}$ & $m^{2}$ & 82,53 & 20,69 & 1707,55 \\
\hline 3 & & Mampostería & & & & 4646,78 \\
\hline 3.1 & 500736 & $\begin{array}{l}\text { Mampostería con burrito largo echado } \\
\text { mortero } 1: 3 \mathrm{e}=2 \mathrm{~cm}\end{array}$ & $\mathrm{~m}^{2}$ & 235,28 & 19,75 & 4646,78 \\
\hline 4 & & $\begin{array}{l}\text { Carpintería metalmecánica - madera } \\
\text { - aluminio y vidrio }\end{array}$ & & & & 1646,24 \\
\hline 4.1 & 500595 & $\begin{array}{l}\text { Suministro e instalación de ventana de } \\
\text { aluminio y vidrio } 6 \mathrm{~mm}\end{array}$ & $\mathrm{~m}^{2}$ & 8,3 & 78,66 & 652,88 \\
\hline 4.2 & 500961 & $\begin{array}{l}\text { Suministro e instalación de puerta } \\
\text { madera } h=2,40 \text { mts. }\end{array}$ & Unidad & 6 & 129,56 & 777,36 \\
\hline 4.3 & 500agn & $\begin{array}{l}\text { Suministro e instalación de ventanas } \\
\text { de madera }\end{array}$ & Unidad & 1 & 216 & 216 \\
\hline 5 & & Cubierta metálica y tumbado & & & & 4428,49 \\
\hline 5.1 & 500657 & Acero estructural a36 & $\mathrm{kg}$ & 789,56 & 3,28 & 2589,76 \\
\hline 5.2 & $500 a 10$ & Cubierta de Galvalume e=0,40mm & $m^{2}$ & 122,91 & 14,96 & 1838,73 \\
\hline 6 & & Instalaciones eléctricas & & & & 2652,18 \\
\hline 6.1 & 500728 & $\begin{array}{l}\text { Suministro e instalación de punto de } \\
\text { iluminación, incluye foco led y Resetón } \\
\text { (tubería } 1 / 2 \text { Emt) }\end{array}$ & Punto & 19 & 44,95 & 854,05 \\
\hline 6.2 & 500ait & $\begin{array}{l}\text { Suministro e instalación de punto de } \\
\text { tomacorriente } 110 \mathrm{v} \text { (tubería } 1 / 2 \mathrm{Emt} \text { ) }\end{array}$ & Punto & 27 & 39,7 & 1071,9 \\
\hline 6.3 & 500865 & $\begin{array}{l}\text { Suministro e instalación de punto } 120 \mathrm{v} \\
\text { para instalación de lámpara de } \\
\text { emergencia, incluye lámpara de } \\
\text { emergencia-led }\end{array}$ & Unidad & 3 & 83,6 & 250,8 \\
\hline
\end{tabular}




\begin{tabular}{|c|c|c|c|c|c|c|}
\hline 6.4 & 500767 & $\begin{array}{l}\text { Suministro e instalación de punto de } \\
\text { tomacorriente } 220 \mathrm{v} \text { (tubería } 1 / 2 \mathrm{Emt} \text { ) }\end{array}$ & Punto & 3 & 62,21 & 186,63 \\
\hline 6.5 & $500 \mathrm{acl}$ & $\begin{array}{l}\text { Suministro e instalación de punto de } \\
\text { toma tv (tubería } 1 / 2 \mathrm{Emt} \text { ) }\end{array}$ & Unidad & 1 & 73,2 & 73,2 \\
\hline 6.6 & 500879 & $\begin{array}{l}\text { Suministro e instalación punto de red, } \\
\text { incluye ductería Emt } 3 / 4^{"}\end{array}$ & Punto & 1 & 168,32 & 168,32 \\
\hline 6.7 & 500672 & $\begin{array}{l}\text { Suministro e instalación de toma } \\
\text { telefónica, servicio }\end{array}$ & Unidad & 1 & 47,28 & 47,28 \\
\hline 7 & & Hidrosanitario & & & & 1591,22 \\
\hline 7.1 & 500064 & $\begin{array}{l}\text { Suministro e instalación de tubería } \\
\text { PVC sanitaria de } 50 \mathrm{~mm}\end{array}$ & $\mathrm{~m}$ & 5 & 6,94 & 34,7 \\
\hline 7.2 & 500065 & $\begin{array}{l}\text { Suministro e instalación de tubería } \\
\text { PVC sanitaria de } 110 \mathrm{~mm}\end{array}$ & $\mathrm{~m}$ & 20 & 10,13 & 202,6 \\
\hline 7.3 & 500645 & $\begin{array}{l}\text { Suministro e instalación de punto de } \\
\text { agua servida de } 50 \mathrm{~mm}\end{array}$ & Punto & 5 & 32,4 & 162 \\
\hline 7.4 & 500063 & $\begin{array}{l}\text { Suministro e instalación de punto de } \\
\text { agua servida de } 110 \mathrm{~mm}\end{array}$ & Punto & 1 & 55,92 & 55,92 \\
\hline 7.5 & 500641 & $\begin{array}{l}\text { Suministro e instalación de tubería de } \\
1 / 2 " \text { de agua potable }\end{array}$ & $\mathrm{m}$ & 21 & 4,16 & 87,36 \\
\hline 7.6 & 500aiu & $\begin{array}{l}\text { Suministro e instalación de tubería de } \\
1 / 2 \text { " de agua potable (agua caliente) }\end{array}$ & $\mathrm{m}$ & 15 & 4,54 & 68,1 \\
\hline 7.7 & 500643 & $\begin{array}{l}\text { Suministro e instalación de punto de } \\
\text { agua potable } 1 / 2 \text { " (incluye corte } 0 \\
\text { picada) }\end{array}$ & Punto & 7 & 25,13 & 175,91 \\
\hline 7.8 & $500 \mathrm{ad} 2$ & $\begin{array}{l}\text { Suministro e instalación de punto de } \\
\text { agua potable 1/2" (caliente) }\end{array}$ & Punto & 2 & 26,12 & 52,24 \\
\hline 7.9 & $500 a 3 z$ & $\begin{array}{l}\text { Suministro e instalación llave de paso } \\
1 / 2 \text { tipo compuerta }\end{array}$ & Unidad & 3 & 27,8 & 83,4 \\
\hline 7.1 & 500897 & $\begin{array}{l}\text { Suministro e instalación de lavabo con } \\
\text { accesorios - incluye llave }\end{array}$ & Unidad & 3 & 108,17 & 324,51 \\
\hline 7.11 & 500 afc & $\begin{array}{l}\text { Suministro e instalación de inodoro } \\
\text { blanco ecológico (incluye llave } \\
\text { angular) }\end{array}$ & Unidad & 1 & 130,42 & 130,42 \\
\hline 7.12 & 500928 & $\begin{array}{l}\text { Suministro e instalación de ducha } \\
\text { incluido llave }\end{array}$ & Unidad & 1 & 34,85 & 34,85 \\
\hline 7.13 & $500 a 5 m$ & $\begin{array}{l}\text { Suministro e instalación de pozo } \\
\text { lavaplatos }\end{array}$ & Unidad & 1 & 138 & 138 \\
\hline 7.14 & 500626 & $\begin{array}{l}\text { Suministro e instalación de llave de } \\
\text { pared con pico alto }\end{array}$ & Unidad & 1 & 41,21 & 41,21 \\
\hline 8 & & Acabados & & & & 3282,64 \\
\hline 8.1 & 500101 & $\begin{array}{l}\text { Suministro e instalación de cerámica } \\
\text { en paredes }\end{array}$ & $\mathrm{m}^{2}$ & 22,46 & 22,36 & 502,21 \\
\hline 8.2 & 500aj5 & $\begin{array}{l}\text { Suministro e instalación de piso de } \\
\text { laurel incluye; pulido de piso y } \\
\text { laqueado. }\end{array}$ & $\mathrm{m}^{2}$ & 82,53 & 24 & 1980,72 \\
\hline 8.3 & $500 a e 3$ & Revestimiento de granito para mesón & $\mathrm{m}$ & 8,6 & 92,99 & 799,71 \\
\hline \multicolumn{6}{|c|}{ Subtotal } & 26852,72 \\
\hline \multirow{2}{*}{\multicolumn{6}{|c|}{$\begin{array}{l}\text { Iva } \\
\text { Total }\end{array}$}} & 3222,33 \\
\hline & & & & & & 30075,05 \\
\hline
\end{tabular}

${ }^{*} \mathrm{~m}$ : metros, kg: kilogramos

Se debe mencionar que, en cada uno de los ítems considerados en este presupuesto, se ha tomado en cuenta el costo equivalente por mano de obra; equipos $y$ materiales, incluyendo el consumo 
de agua asociado a los ítems correspondientes.

Por consiguiente, el total reflejado en la tabla 1 (\$30075,05 dólares americanos incluido IVA) equivale al costo total requerido para ejecutar la construcción del diseño de vivienda de interés social con mampostería confinada en ladrillo, propuesto en la presente investigación.

Por su parte, considerando que el sueldo básico unificado en el Ecuador para el año 2021 es $\$ 400$, la ejecución y construcción del sistema estructural propuesto representaría un total de 75,19 sueldos básicos.

Carrillo et al. (2015) realizaron una evaluación de varios diseños estructurales de viviendas con mampostería confinada y muros de concreto reforzados con malla electrosoldada o con fibras de acero, a partir del precio unitario de materiales, equipos y mano de obra. Por su parte, Echeverry et al. (2000) realizaron comparaciones entre distintos prototipos de viviendas de interés social, proponiendo el sistema de mampostería reforzada como el más viable desde el punto de vista financiero.

Es necesario considerar que la vivienda de interés social propuesta en la presente investigación se encuentra diseñada en una superficie de 82 $\mathrm{m}^{2}$, lo cual le otorga múltiples ventajas, dado que los demás diseños ocupan una superficie de construcción menor. Por ende, la relación costo/área de la construcción refleja $\$ 366,77$ por cada $\mathrm{m}^{2}$, representando una ratio inferior al planteado en otras propuestas de VIS. Cabe señalar que el sistema convencional se encuentra en un ratio que oscila entre $\quad \$ 500-600 / \mathrm{m}^{2}$, siendo evidentes las ventajas de la presente propuesta. También se mencionan los beneficios de este diseño, en base a criterios técnicos de las normas de construcción que demuestran su factibilidad desde el plano estructural y sismorresistente (Macias et al., 2017; Valente et al., 2019).

Se debe considerar el desempeño sísmico en el diseño estructural de vivienda de interés social. Por consiguiente, Carrillo \& Alcocer (2013) concluyen que es factible proporcionar en viviendas de 
concreto las cualidades sismoresistentes requeridas para aportar seguridad frente a movimientos telúricos, en función de los materiales empleados, la geometría estructural y la demanda sísmica de cada uno de los muros.

\section{Conclusiones}

En dependencia del presupuesto establecido y los análisis realizados, se estableció que la vivienda de interés social con mampostería confinada en ladrillo presenta un sistema estructural favorable para su aplicación en el medio local de Manta y de la misma forma puede extrapolarse a otros cantones de la provincia de Manabí y del Ecuador.

Cabe señalar que en la presente investigación se planteó un diseño estructural con las dimensiones y presupuestos que se encuentran estandarizados en las normas de construcción y en dependencia de la información aportada por ferreterías y empresas constructoras, resaltando que el área de construcción equivale a 82 $\mathrm{m}^{2}$, demostrando de esta forma que la relación costo/superficie $\left(\$ 366,77 / \mathrm{m}^{2}\right)$ de la presente propuesta de VIS es inferior a los ratios de los sistemas convencionales comúnmente empleados en el Ecuador.

\section{Bibliografía}

Bamba, J. C. (2016). La vivienda social en Guayaquil (19401970): procesos de transformación en los espacios colectivos. Arquitecturas del Sur, 34(49), 66-79.

BID. (2014). Urbanización rápida y desarrollo: cumbre de América Latina y China. Nueva York: Banco Interamericano de Desarrollo.

Cárdenas, R. \& Macancela, E. (2018). Análisis comparativo del sistema muros portantes de hormigón frente al sistema convencional de mampostería confinada en una vivienda tipo social. Tesis de pregrado. Facultad de Ingeniería, Universidad de Cuenca. Disponible en: http://dspace.ucuenca.edu.ec /handle/123456789/30280

Carrillo, J., \& Alcocer, S. M. (2013). Shear strength of reinforced concrete walls for seismic design of low-rise housing. $\mathrm{ACl}$ Structural Journal, 110(3), 415.

Carrillo, J., Aperador, W., \& Echeverri, F. (2015). Evaluación de los costos de construcción de sistemas estructurales para viviendas de baja altura y de interés 
social.

Ingeniería, investigación y tecnología, 16(4), 479-490. DOI: https://doi.org/10.1016/j.riit.20 15.09.001

Echeverry, D., Sarria, A., Alarcón, L., Torres, W., Prieto, J., \& Flórez, J. (2020). Vivienda de interés social: Inventario de sistemas constructivos, Departamento de Ingeniería Civil y Ambiental y Universidad de los Andes, Metro Vivienda, Bogotá, 2000.

INEC. (2010). Ecuador en Cifras. Quito: Instituto Nacional de Estadísticas y Censos. Obtenido de: http://ecuadorencifras.gob.ec/ resultados

Macias, J., Iturburu, L., Rodriguez, C., Agdas, D., Boero, A., \& Soriano, G. (2017). Embodied and operational energy assessment of different construction methods employed on social interest dwellings in Ecuador. Energy and Buildings, 151, 107-120. https://doi.org/10.1016/j.enbui ld.2017.06.016

Ocampo, J. S. (2017). Vivienda industrializada: antecedentes en el mundo y propuesta al déficit de vivienda social en Colombia. Cuadernos de Vivienda y Urbanismo, 10(20), 79-96.

DOI: https://doi.org/10.11144/Javer iana.cvu10-20.viam

Pérez-Pérez, A. L. (2016). El diseño de la vivienda de interés social. La satisfacción de las necesidades y expectativas del usuario. Revista de Arquitectura, 18(1), 67-75. Doi:10.14718/RevArq.2016.1

\subsection{7}

Valente, M., Milani, G., Grande, E., \& Formisano, A. (2019). Historical masonry building aggregates: advanced numerical insight for an effective seismic assessment on two row housing compounds. Engineering Structures, 190, 360-379. https://doi.org/10.1016/j.engst ruct.2019.04.025 